\title{
Protein short loop prediction in terms of a structural alphabet
}

\author{
Manoj Tyagi $^{12}$, Aurélie Bornot ${ }^{23}$, Bernard Offmann ${ }^{134}$, Alexandre G. De Brevern ${ }^{2}{ }^{*}$
}

1 LBGM, Laboratoire de Biochimie et Génétique Moléculaire Université de la Réunion, BP 7151, 15 avenue René Cassin, 97715 Saint Denis Messag Cedex 09, La Réunion,FR

${ }^{2}$ Bioinformatique génomique et moléculaire INSERM : U726, Université Paris-Diderot - Paris VII , 2, Place Jussieu 75251 PARIS CEDEX $05, F R$

3 Protéines de la membrane érythrocytaire et homologues non-érythroides INSERM : U665, Université Paris-Diderot - Paris VII , INTS 6, Rue Alexandre Cabanel 75739 PARIS CEDEX 15,FR

4 PEACCEL PEACCEL, Technopole, Saint-Denis de la réunion,IN

* Correspondence should be adressed to: Alexandre De Brevern <alexandre.debrevern@univ-paris-diderot.fr >

\begin{abstract}
Loops connect regular secondary structures. In many instances, they are known to play crucial biological roles. To bypass the limitation of secondary structure description, we previously defined a structural alphabet composed of 16 structural prototypes, called Protein Blocks (PBs). It leads to an accurate description of every region of 3D protein backbones and have been used in local structure prediction.
\end{abstract}

In the present study, we used our structural alphabet to predict the loops connecting two repetitive structures. Thus, we showed the interest to take into account the flanking regions, leading to prediction rate improvement up to $19.8 \%$, but we also underline the sensitivity of such an approach. This research can be used to propose different structures for the loops and to probe and sample their flexibility. It is a useful tool for ab initio loop prediction and leads to insights to flexible docking approach.

MESH Keywords Computer Simulation ; Databases, Protein ; Protein Structure, Secondary ; Proteins ; chemistry ; metabolism

Author Keywords local protein structure ; protein loops ; secondary structure ; protein function ; bioinformatics ; biophysics.

\section{Introduction}

The knowledge of the three-dimensional (3D) structures of proteins contributes to understand their biological functions. Protein 3D structures are often described as a succession of repetitive secondary structures (mainly a-helices and $\beta$-sheets (Pauling and Corey, 1951 ; Pauling et al., 1951 )). This mono-dimensional description helps to simplify coarsely this 3D information.

Nevertheless, the secondary structures description focuses only on two kinds of regular local structures that compose only a part of protein backbone. The remaining residues are only assigned if they can be associated with some particular structures such as the $\beta$-turns ( Bornot and de Brevern, 2006 ; Wang et al., 2006 ). It has led some scientific teams to develop local protein structure libraries that (i) are able to approximate all (or almost all) the local protein structures and (ii) do not make use of the classical secondary structures assignments. These libraries lead to the categorization of 3D structures without any a priori into small prototypes that are specific to local folds found in proteins. The complete set of local structure prototypes defines a structural alphabet (Karchin et al., 2003 ; Offmann et al., 2007 ). The number of libraries or structural alphabet is important, e.g . (Martin et al., 2008 ; Sander et al., 2006 ; Tung et al., 2007 ).

Our structural alphabet is composed of 16 mean protein fragments of 5 residues in length, called Protein Blocks (PBs, see Figure 1 ) ( de Brevern et al., 2000 ). They have been used both to describe the 3D protein backbones (de Brevern, 2005 ) and to perform a local structure prediction (de Brevern et al., 2000 ; de Brevern et al., 2004 ; Dong et al., 2008 ; Etchebest et al., 2005 ; Zimmermann and Hansmann, 2008 ). PBs have proven their efficiency in the description and the prediction of long fragments (Benros et al., 2009 ; Benros et al., 2006 ; Bornot et al., 2009 ; de Brevern and Hazout, 2003 ; de Brevern et al., 2002 ; de Brevern et al., 2007 ), to compare protein structures (Tyagi et al., 2008 ; Tyagi et al., 2006a ; Tyagi et al., 2006b ), to build globular (Dong et al., 2007 ) and transmembrane protein structures (de Brevern, 2009 ; de Brevern et al., 2009 ; de Brevern et al., 2005 ), to define a reduced amino acid alphabet dedicated to mutation design (Etchebest et al., 2007 ), to design peptides (Thomas et al., 2006 ), to define binding site signatures (Dudev and Lim, 2007 ) or to analyze protein contacts (Faure et al., 2009 ). The features of this alphabet have been compared with those of 8 other structural alphabets showing clearly that our PB alphabet is highly informative, with the best predictive ability of those tested (Karchin et al., 2003 ).

In this paper, we focus on the prediction of short loops from sequence. Loop prediction is one of the major drawbacks of homology modeling methods even if loops play crucial biological role. Loop prediction is frequently performed after the positioning of repetitive secondary structures. Current protocols are based on the sampling of the conformational space of the loop fragment and the scoring of the corresponding sampled conformations, but do not perform well for loops more than 10 residues (Zhu et al., 2006 ). 
Prediction using a structural alphabet is an efficient way to predict loop structures. Indeed, as the PBs are overlapping and more precise than secondary structure, a small modification of PB series has a fewer consequence in comparison to a change observed in secondary structure assignment. Moreover, PBs are successful to align structural homologues even in difficult case with very divergent loops (Tyagi et al., 2008 ; Tyagi et al., 2006a ). Following a previous research (Fourrier et al., 2004 ), we described short loops that connect two repetitive structures using our structural alphabet, and performed local loop structure predictions from the amino acid sequence. In this study we assessed the importance of a priori knowledge of the flanking regions and of sequence identity of the protein databank on the loop prediction.

\section{Methods}

\section{Data sets}

10 non-redundant protein databanks have been used in this study. They are based on the PISCES database (Wang and Dunbrack, 2005 ) and represents between 162,830 (namely DB0) and 1,572,412 residues (namely DB8, see supplementary data I ). They are available at http://www.dsimb.inserm.fr/ debrevern/DOWN/DB/new . The sets are defined as containing no more than $\mathrm{x} \%$ pairwise sequence identity with $\mathrm{x}$ ranging from 20 to $90 \%$. The selected chains have X-ray crystallographic resolutions less than $1.6 \AA$ with an R-factor less than 0.25 or less than $2.5 \AA$ with an R-factor less than 1.0. Each chain was carefully examined with geometric criteria to avoid bias from zones with missing density (Tyagi et al., 2009 ).

\section{Protein Blocks \& short loop description}

Protein Blocks (PBs) correspond to a set of 16 local prototypes, labeled from a to p (cf. Figure 1 ), of 5 residues length based on $\Phi, \Psi$ dihedral angles description. They were obtained by an unsupervised classifier similar to Kohonen Maps (Kohonen, 1982 ) and Hidden Markov Models (Rabiner, 1989 ). The PBs $\mathrm{m}$ and d can be roughly described as prototypes for central $\alpha$-helix and central $\beta$-strand, respectively. PBs a through c primarily represent $\beta$-strand $\mathrm{N}$-caps and PBs e and $\mathrm{f}, \mathrm{C}$-caps; PBs g through $\mathrm{j}$ are specific to coils, PBs $\mathrm{k}$ and 1 to $\alpha$-helix N-caps, and PBs $n$ through $p$ to $\mathrm{C}$-caps. This structural alphabet allows a reasonable approximation of local protein $3 \mathrm{D}$ structures (de Brevern et al., 2000 ) with a root mean square deviation (rmsd ) now evaluated at $0.42 \AA$ (de Brevern, 2005 ). PBs (de Brevern, 2005 ) have been assigned using in-house software (available at http://www.dsimb.inserm.fr/DOWN/LECT/ ), it follows similar rules to assignment done by PBE web server (http://bioinformatics.univ-reunion.fr/PBE/ ) (Tyagi et al., 2006b ). We have defined the short loops as PB series of length 2 to 6 . They represent the most frequent loop lengths. These series must be composed of non-repetitive PBs, i.e. all the PBs except PBs d and $\mathrm{m}$. They must have flanking regions composed of series of PBs mm and/or dd (Fourrier et al., 2004 ).

\section{Prediction}

In a strategy of structure prediction from sequence (de Brevern et al., 2000 ; de Brevern et al., 2004 ; Etchebest et al., 2005 ), we must compute for a given sequence window $\mathrm{S}_{\mathrm{aa}}=\left\{\mathrm{aa}_{-_{\mathrm{w}}}, \ldots, \mathrm{aa}_{0}, \ldots, \mathrm{aa}_{\mathrm{+}_{\mathrm{w}}}\right\}$, the probability of observing a given protein block $\mathrm{PB}{ }_{\mathrm{x}}$, i.e. $\mathrm{P}\left(\mathrm{PB}_{\mathrm{x}} \mid\right.$ $\mathrm{S}_{\mathrm{aa}}$ ). For this purpose, each PB is associated with an occurrence matrix of dimension $1 \times 20$ centered upon the PB, with $1=2 \mathrm{w}+1$ (in the study, w =7). Using the Bayes theorem to compute this a posteriori probability $P\left(\mathrm{~PB}_{\mathrm{x}} \mid \mathrm{S}_{\mathrm{aa}}\right)$ from the a priori probability $\mathrm{P}\left(\mathrm{S}_{\mathrm{aa}} \mid \mathrm{PB}_{\mathrm{x}}\right)$ deduced from the occurrence matrix allows us to define the odds score $\mathrm{R}_{\mathrm{x}}$ :

$$
R_{\chi}=\prod_{j=-w}^{j=+w} \frac{P\left(a a_{j}=i \mid P B_{\chi}\right)}{P\left(a a_{j}=i \mid D B\right)}
$$

The highest score $\mathrm{R}_{\mathrm{x}}$ corresponds to the most probable PB (de Brevern et al., 2000 ; de Brevern et al., 2002 ; de Brevern et al., 2007 ; de Brevern et al., 2004 ; Etchebest et al., 2005 ; Fourrier et al., 2004 ). The $\mathrm{Q}_{16}$ value computed is the total number of true predicted PBs over the total number of predicted PBs. We also computed a $Q_{14}$ value, specific for loops, i.e. the PB $\mathrm{m}$ and $\mathrm{d}$ are not taken into account in the accuracy rate computation (Fourrier et al., 2004 ).

Thus different predictions have been done: (i) one general prediction done for comparison purpose, it gives the $Q_{16}$ and $Q_{14}$ values, (ii) a specific prediction of only short loops, it gives a $Q_{14}$ short values and (iii) specific prediction of only short loops $\alpha-\alpha, \alpha-\beta, \beta-\alpha$ and $\beta-\beta$, it gives a $\mathrm{Q}_{14}$ short values for each kinds of short loops.

\section{Results \& Discussion}

Prediction of structural alphabets could be strongly dependant of the selection of the training and validation set (Etchebest et al., 2005 ). This feature is amplified in the case when the letters of the structural alphabets have low frequency, e.g . $\pi$-helix (Wang et al., 2006 ), type VI $\beta$-turns (Fuchs and Alix, 2005 ) or PB b (de Brevern, 2005 ). Specific learning and validation were performed (i) for all the PBs (for comparison purpose), (ii) a specific research focusing on all the protein loops, and finally (iii) for each kinds of loops, i.e . $\alpha$ - $\alpha$, $\alpha-\beta, \beta-$ 
$\alpha$ and $\beta-\beta$. Protein databanks have been split randomly into $2 / 3$ of the proteins used for the training step and $1 / 3$ for the validation step. We have done large number of independent simulations for optimizing the prediction rates, i.e . for each simulation the proteins used for the training and the validation sets are different.

\section{Global prediction rates}

Figure 1 shows 1000 independent simulations performed with the databank DB0 (887 protein chains, see supplementary material I ). This Figure shows the importance of sampling. The difference between average and best prediction values is $1 \%$ for the prediction rate $\mathrm{Q}$ ${ }_{16}$ and increases with the diminution of the databank, e.g . this difference equals $8 \%$ for the prediction of short loops connecting $\alpha$-helix to $\beta$-strand. The best prediction rates for each of the PBs have been summarized in Table 1 . These results highlight the interest to perform specific prediction for each kind of short loops; a specific learning with the a priori knowledge of the flanking regions improves the prediction rate. Nonetheless, this improvement is not uniform for all the short loop kinds.

From a correct $\mathrm{Q}_{16}$ value of $35.8 \%$, a prediction specific to loops, i.e. $\mathrm{Q}_{14}$, reaches $37.5 \%(+1.7 \%)$, and to $43.2 \%(+5.7 \%)$, if only the short loops are used. An increase ranging from $0.7 \%$ to $19.0 \%$ was observed for the 4 types of short loops.

We thus confirm our previous results, but also underline the importance of shuffling the proteins between validation and learning set (as we have also shown in (Etchebest et al., 2005 )). It allows to improve all the prediction rates in comparison to (Fourrier et al., 2004 ), thus $\mathrm{Q}_{16}$ goes from 35.2 to $35.8 \%$ and the corresponding $\mathrm{Q}_{14}$ from 36.0 to $37.4 \%$ highlighting the quality of prediction, i.e . this improvement is not due only to PBs $\mathrm{m}$ and $\mathrm{d}$, the repetitive PBs. In the same way, $\mathrm{Q}_{14}$ for the short loops also increases from $\mathrm{Q}_{14}=42.3$ to $43.2 \%$. For the 4 types of short loops, the difference between average and best prediction rates bypasses $5 \%$ in all the cases.

This global improvement is also associated with an increase of the over - training, i.e . a prediction largely better with the learning set than with the validation set. For the prediction of all residues, the over - training rate is less than $1 \%$ on average; it increases to $4 \%$ for the short loops and increases again for the short loop types, this value can reach $20 \%$ and more. Nevertheless, a strong linear correlation exists $(r>0.95)$ between the prediction rate and the over-training. The better is the prediction rate, the lower is the over - training. Thus interestingly, the selected predictions are associated with the lower over - training rates.

These values represent only a compatibility between $\left(2 \mathrm{D}^{\prime}\right)$ representation, i.e. true PBs and predicted PBs. So, we also assessed the quality of local structure approximation using $\mathrm{C}_{\alpha}$ root mean square deviation (rmsd ) measure. We superimposed all the true PBs to the local protein structure, even the good PB, in order to compute the expected rmsd random values and we found they are always between 1.95 and $2.00 \AA$ A. The percentage of predicted PBs better than random value corresponds to $64 \%$ and $66 \%$ for the loops and short loops prediction respectively. These values increase with the specialisation of short loops with prediction rates equal to $68 \%$ for $\beta-\alpha, 74 \%$ for $\beta-\beta$ , $76 \%$ for $\alpha-\alpha$ and $82 \%$ for $\alpha-\beta$.

\section{PBs prediction rates}

Improvements of prediction rates for the short loops are due to different PBs. For the all short loops, only two PBs clearly lost prediction rate, i.e. PBs $j$ and 1 . The most frequent PBs have often very good prediction rate. For the $\alpha-\alpha$ short loops, only two PBs present a decreased prediction rate (PBs a and h), for $\alpha-\beta, 5$ (PBs f , h, i , k and 1 ), for $\beta-\alpha 5$ (PBs a , c , h, i and j ) and 3 for $\beta-\beta$ (PBs h , $\mathrm{n}$ and o ). As most of these PBs have very low frequencies (mainly less than $5 \%$ of the PBs in their kind of loops), these losses of their prediction rate is not significant. We can note that PBs h never obtains a prediction gain with this approach, at the opposite, the prediction of PBs b and $\mathrm{p}$, which are related to some $\beta$-strand $\mathrm{N}$ capping regions is improved in each case.

\section{Influence of protein databanks}

All the prediction rates presented bellow belongs to the smallest databank (DB0). Interestingly these prediction rates do not increase with the growth of the protein databank, i.e . databanks with more redundancy. The best prediction rates remain always near identical, thus the similarity sequence rate does not improve this prediction approach based on a single sequence. Nonetheless, the over-training rate decreases greatly with the size of the databank, i.e . for ( $80 \%$ of sequence identity, resolution better than $2.5 \AA$ and R-factor less than 1.00$)$, the biggest one, the over-training rate is less than $2 \%$ thanks to the redundancy of the protein databank.

In the same way, it must be noticed that it does not change the kind of amino occurrence matrices, i.e . no new amino acid specificities are observed or disappeared (data not shown). This fact underlines that Bayesian prediction is an interesting tool to predict and analyze the local protein structures, we expect to use in future more sophisticated methods less dependant on the size of databank. One advantage of such an approach is that it enables us to compute the most significant series of PBs and from this information propose alternative 3D candidate structures as we have shown previously (Fourrier et al., 2004 ).

\section{Conclusion}


Prediction of short loops taking into account of the flanking regions clearly improves the quality of prediction, with only limited effect on $\beta$ to a short loops, but near $20 \%$ of gain for a to a short loops. The use of numerous non-redundant databanks show: (i) global prediction rates remain equivalent for all the protein databank used, with no influence of the sequence identity or quality of crystallographic resolution and (ii) the importance of over - training, this last diminished greatly with the increase of the protein databank size.

Though sometimes the prediction rates of each PB could vary a little, most of the time it is less than $5 \%$. We can notice that four PBs are associated with more variation of their prediction rates, interestingly their frequencies in the most redundant databanks are decreased in regards to the lower redundant databanks. Thus, a slight statistical bias is observed.

In this field, prediction of loops using a structural alphabet is an interesting tool. Instead of describing entire loops; our approach predicts each position in the loops locally. To take into account the flanking regions of the loops greatly improve the prediction rates. As nearly all protein fragments can be can be observed in the Protein DataBank (Balamurugan et al., 2005 ; Balamurugan et al., 2006 ; Du et al., 2003 ), this approach can be used to propose many different structures for the loops and to probe and sample their flexibility, and, is a useful tool in ab initio loop prediction.

\section{Ackowledgements:}

This work was supported by grants from the Ministère de la Recherche, Université Paris Diderot - Paris 7, Université de Saint-Denis de la Ré union and the French Institute for Health and Medical Care (INSERM). MT had a grant from the Conseil Régional de Réunion and AB has a grant from Ministère de la Recherche.

\section{References:}

- Balamurugan B , Samaya Mohan K, Ramesh J , Roshan MN , Sumathi K, Sekar K . 2005 ; SSEP-2.0: Secondary Structural Elements of Proteins . Acta Crystallogr D Biol Crystallogr . $61: 634-636$

- Balamurugan B , Roshan MN , Michael D , Ambaree M, Divya S , Keerthana H, Seemanthini M, Sekar K . 2006 ; SMS: sequence, motif and structure--a database on the structural rigidity of peptide fragments in non-redundant proteins . In Silico Biol . 6:229-235

- Benros C, de Brevern AG, Hazout S . 2009 ; Analyzing the sequence-structure relationship of a library of local structural prototypes . J Theor Biol . 256 : 215 - 226

- Benros C, de Brevern AG, Etchebest C, Hazout S . 2006 ; Assessing a novel approach for predicting local 3D protein structures from sequence . Proteins . 62 : 865 - 880

- Bornot A, de Brevern AG . 2006 ; Protein beta-turn assignments . Bioinformation . $1: 153$ - 155

- Bornot A, Etchebest C, de Brevern AG . 2009; A new prediction strategy for long local protein structures using an original description . Proteins

- de Brevern AG . 2005 ; New assessment of a structural alphabet. In Silico Biol . $5: 283$ - 289

- de Brevern AG . 2009 ; New opportunities to fight against infectious diseases and to identify pertinent drug targets with novel methodologies . Infect Disord Drug Targets . 9 : $246-247$

- de Brevern AG, Hazout S . 2003 ; 'Hybrid protein model' for optimally defining 3D protein structure fragments . Bioinformatics . 19 : 345 - 353

- de Brevern AG, Etchebest C, Hazout S . 2000 ; Bayesian probabilistic approach for predicting backbone structures in terms of protein blocks . Proteins . 41 : 271 - 287

- de Brevern AG, Valadie H, Hazout S , Etchebest C . 2002 ; Extension of a local backbone description using a structural alphabet: a new approach to the sequence-structure relationship . Protein Sci . $11: 2871-2886$

- de Brevern AG, Etchebest C, Benros C, Hazout S . 2007 ; "Pinning strategy": a novel approach for predicting the backbone structure in terms of protein blocks from sequence J Biosci . $32: 51-70$

- de Brevern AG, Autin L, Colin Y, Bertrand O, Etchebest C . 2009 ; In silico studies on DARC . Infect Disord Drug Targets . 9 : 289 - 303

- de Brevern AG, Benros C, Gautier R, Valadie H, Hazout S, Etchebest C. 2004 ; Local backbone structure prediction of proteins . In Silico Biol . 4 : 381 - 386

- de Brevern AG, Wong H, Tournamille C, Colin Y, Le Van Kim C, Etchebest C . 2005 ; A structural model of a seven-transmembrane helix receptor: the Duffy antigen/receptor for chemokine (DARC) . Biochim Biophys Acta . 1724: 288 - 306

- DeLano WLT . 2002 ; The PyMOL Molecular Graphics System DeLano Scientific . San Carlos ; CA, USA http://www.pymol.org

- Dong Q, Wang X, Lin L . 2008 ; Prediction of protein local structures and folding fragments based on building-block library . Proteins . 72 : 353 - 366

- Dong QW, Wang XL, Lin L . 2007 ; Methods for optimizing the structure alphabet sequences of proteins . Comput Biol Med . 37 : 1610 - 1616

- Du P , Andrec M, Levy RM . 2003 ; Have we seen all structures corresponding to short protein fragments in the Protein Data Bank? An update . Protein Eng . 16 : 407 - 414

- Dudev M , Lim C . 2007 ; Discovering structural motifs using a structural alphabet: application to magnesium-binding sites . BMC Bioinformatics . 8 : 106 -

- Etchebest C, Benros C, Hazout S, de Brevern AG . 2005 ; A structural alphabet for local protein structures: improved prediction methods . Proteins . 59 : 810 - 827

- Etchebest C, Benros C, Bornot A, Camproux AC, de Brevern AG . 2007 ; A reduced amino acid alphabet for understanding and designing protein adaptation to mutation . Eur Biophys J . $36: 1059-1069$

- Faure G, Bornot A, de Brevern AG . 2009 ; Analysis of protein contacts into Protein Units . Biochimie . 91 : 876 - 887

- Fourrier L, Benros C , de Brevern AG . 2004 ; Use of a structural alphabet for analysis of short loops connecting repetitive structures . BMC Bioinformatics . 5 : 58 -

- Fuchs PF , Alix AJ . 2005 ; High accuracy prediction of beta-turns and their types using propensities and multiple alignments . Proteins . 59 : 828 - 839

- Karchin R , Cline M , Mandel-Gutfreund Y, Karplus K . 2003 ; Hidden Markov models that use predicted local structure for fold recognition: alphabets of backbone geometry Proteins . $51: 504-514$

- Kohonen T . 1982 ; Self-organized formation of topologically correct feature maps . Biol Cybern . 43 : 59 - 69

- Martin J , de Brevern AG, Camproux AC . 2008 ; In silico local structure approach: a case study on outer membrane proteins . Proteins . 71 : 92 - 109

- Offmann B , Tyagi M, de Brevern AG . 2007 ; Local Protein Structures . Current Bioinformatics . 3 : 165 - 202

- Pauling L, Corey RB . 1951 ; The pleated sheet, a new layer configuration of polypeptide chains . Proc Natl Acad Sci U S A . 37 : 251 - 256

- Pauling L, Corey RB, Branson HR . 1951 ; The structure of proteins; two hydrogen-bonded helical configurations of the polypeptide chain . Proc Natl Acad Sci U S A . 37 : $205-211$

- Rabiner LR . 1989; A tutorial on hidden Markov models and selected application in speech recognition . Proceedings of the IEEE . 77 : 257 - 286

- Sander O, Sommer I, Lengauer T . 2006 ; Local protein structure prediction using discriminative models . BMC Bioinformatics . 7 : 14 -

- Thomas A, Deshayes S, Decaffmeyer M, Van Eyck MH, Charloteaux B , Brasseur R . 2006 ; Prediction of peptide structure: how far are we? . Proteins . 65 : 889 - 897

- Tung CH , Huang JW , Yang JM . 2007 ; Kappa-alpha plot derived structural alphabet and BLOSUM-like substitution matrix for rapid search of protein structure database . Genome Biol . $8:$ R31 -

- Tyagi M, de Brevern AG, Srinivasan N, Offmann B . 2008 ; Protein structure mining using a structural alphabet . Proteins . 71 : 920 - 937

- Tyagi M, Bornot A, Offmann B, de Brevern AG . 2009; Analysis of loop boundaries using different local structure assignment methods . Protein Sci . in press 
- Tyagi M, Gowri VS, Srinivasan N, de Brevern AG, Offmann B . 2006a ; A substitution matrix for structural alphabet based on structural alignment of homologous proteins and its applications. Proteins . $65: 32-39$

- Tyagi M, Sharma P, Swamy CS, Cadet F, Srinivasan N, de Brevern AG, Offmann B . 2006b ; Protein Block Expert (PBE): a web-based protein structure analysis server using a structural alphabet . Nucleic Acids Res . 34 : W119 - 123

- Wang G , Dunbrack RL Jr . 2005 ; PISCES: recent improvements to a PDB sequence culling server . Nucleic Acids Res . 33 : W94 - 98

- Wang Y, Xue ZD , Shi XH , Xu J . 2006 ; Prediction of pi-turns in proteins using PSI-BLAST profiles and secondary structure information . Biochem Biophys Res Commun . $347: 574-580$

- Zhu K, Pincus DL, Zhao S, Friesner RA . 2006 ; Long loop prediction using the protein local optimization program . Proteins . 65 : 438 - 452

- Zimmermann O , Hansmann UH . 2008 ; LOCUSTRA: accurate prediction of local protein structure using a two-layer support vector machine approach . J Chem Inf Model . $48: 1903-1908$

\section{Figure 1}

The 16 Protein Blocks

From left to right and top to bottom, PyMol (DeLano, 2002 ) images of the 16 Protein Blocks of the structural alphabet (de Brevern, 2005 ; de Brevern et al., 2000 ). Each prototype is five residues in length and corresponds to eight dihedral angles $(\phi, \psi)$. The PBs $\mathrm{m}$ and $\mathrm{d}$ can be roughly described as prototypes for the central $\alpha$-helix and the central $\beta$-strand, respectively. For each PB, the N cap extremity is on the left and the C-cap on the right.

(a)

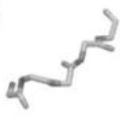

(e)

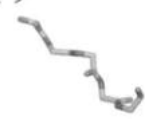

(i)

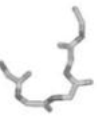

(m)

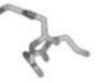

(b)

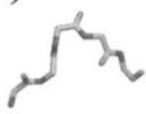

(f)<smiles>CCCCCCC(C)C</smiles>

(j)

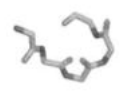

(n)

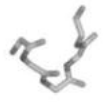

(c)

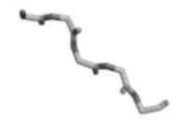

(g)

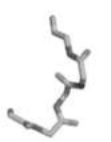

(k)

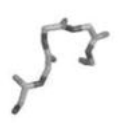

(o)<smiles>CCCCCCCCCCCC</smiles>

(d)

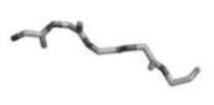

(h)

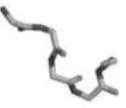

(I)

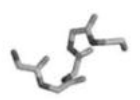

(p)

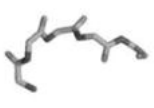

\section{Figure 2}

Distribution of prediction rates

Are given the prediction rate for the classical prediction $\left(\mathrm{Q}_{16}\right)$, a prediction dedicated to the loops $\left(\mathrm{Q}_{14}\right)$, to the short loops $\left(\mathrm{Q}_{14}\right.$ short loops $)$, to the short loops connecting $\alpha$-helix to $\alpha$-helix ( $\alpha \alpha$ ), $\alpha$-helix to $\beta$-strand $(\alpha \beta), \beta$-strand to $\alpha$-helix ( $\beta \alpha$ ) and $\beta$-strand to $\alpha$-helix ( $\beta \beta$ ). The grey dots correspond to the 1000 independent simulation prediction rates, the blue dots to the average prediction value of the prediction rates and the red ones to the best prediction rates.

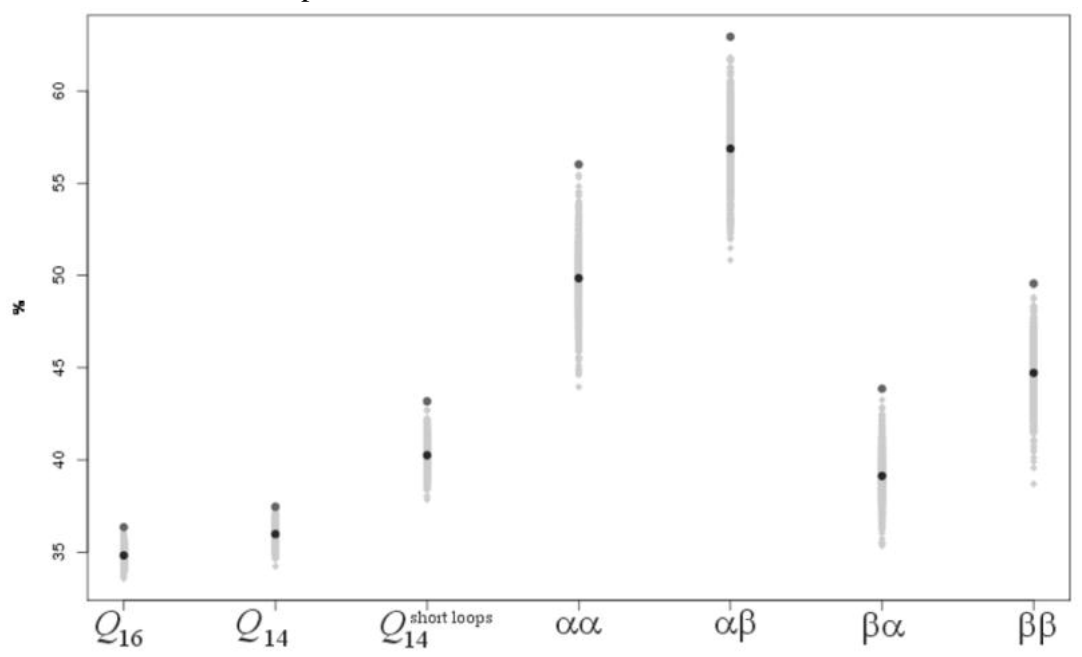




\section{Table 1}

The different PBs' predictions

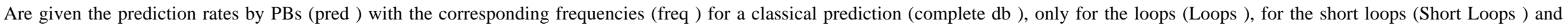

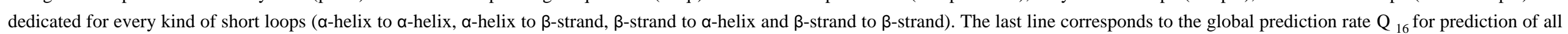
residues and $\mathrm{Q}_{14}$ only for short loops. Results are based on DB0.

\begin{tabular}{|c|c|c|c|c|c|c|c|c|c|c|c|c|c|c|}
\hline \multirow[b]{2}{*}{ PBs } & \multicolumn{2}{|c|}{ complete db. } & \multicolumn{2}{|c|}{ Loops } & \multicolumn{2}{|c|}{ Short loops } & \multicolumn{2}{|r|}{$\alpha \alpha$} & \multicolumn{2}{|r|}{$\alpha \beta$} & \multicolumn{2}{|c|}{$\beta \alpha$} & \multicolumn{2}{|r|}{$\beta \beta$} \\
\hline & pred. & freq. & pred. & freq. & pred. & freq. & pred. & freq. & pred. & freq. & pred. & freq. & pred. & freq. \\
\hline $\mathrm{a}$ & 60.28 & 3.87 & 62.22 & 8.48 & 68.11 & 9.07 & 63.10 & 7.50 & 80.51 & 16.29 & 27.78 & 1.39 & 72.00 & 12.33 \\
\hline $\mathrm{b}$ & 14.03 & 4.02 & 11.06 & 6.48 & 17.92 & 4.78 & 21.74 & 2.05 & 23.08 & 3.26 & 33.66 & 7.78 & 20.41 & 5.37 \\
\hline $\mathrm{c}$ & 27.75 & 7.64 & 26.13 & 10.12 & 32.79 & 7.31 & 35.82 & 11.96 & 46.51 & 10.78 & 28.12 & 2.46 & 40.71 & 6.19 \\
\hline d & 28.50 & 16.37 & 24.69 & 4.78 & & & & & & & & & & \\
\hline $\mathrm{e}$ & 39.98 & 2.18 & 37.39 & 4.90 & 47.27 & 4.71 & & & & & 55.71 & 5.39 & 50.00 & 10.74 \\
\hline $\mathrm{f}$ & 29.65 & 6.44 & 36.40 & 13.60 & 40.94 & 15.60 & 70.44 & 14.20 & 0.00 & 1.00 & 48.16 & 33.41 & 54.72 & 13.92 \\
\hline $\mathrm{h}$ & 44.39 & 2.08 & 43.82 & 5.17 & 50.18 & 4.81 & 33.33 & 1.34 & 33.33 & 2.26 & 46.88 & 4.93 & 48.55 & 9.48 \\
\hline $\mathrm{i}$ & 36.22 & 1.64 & 37.41 & 3.99 & 45.88 & 3.32 & & & 37.04 & 2.26 & 22.22 & 2.08 & 49.19 & 6.79 \\
\hline $\mathrm{i}$ & 56.98 & 0.77 & 44.98 & 1.50 & 29.17 & 1.23 & & & & & 16.67 & 2.31 & 37.04 & 1.48 \\
\hline $\mathrm{k}$ & 38.66 & 5.33 & 42.05 & 12.55 & 37.15 & 15.85 & 64.81 & 19.29 & 20.00 & 2.09 & 54.75 & 30.79 & 52.91 & 11.29 \\
\hline 1 & 38.85 & 5.40 & 24.48 & 4.63 & 16.32 & 3.25 & 22.22 & 0.80 & 12.50 & 1.34 & 19.64 & 4.31 & 18.18 & 3.62 \\
\hline $\mathrm{m}$ & 38.19 & 34.36 & 17.26 & 2.02 & & & & & & & & & & \\
\hline $\mathrm{n}$ & 51.23 & 2.28 & 54.61 & 4.90 & 59.35 & 6.87 & 65.29 & 10.80 & 76.92 & 17.38 & & & 26.83 & 2.25 \\
\hline o & 49.05 & 2.95 & 52.58 & 6.66 & 55.07 & 8.95 & 63.64 & 11.79 & 74.16 & 17.46 & & & 53.66 & 6.74 \\
\hline $\mathrm{P}$ & 33.39 & 3.56 & 35.76 & 7.85 & 43.17 & 10.27 & 57.04 & 12.68 & 64.84 & 21.39 & & & 53.50 & 8.60 \\
\hline $\mathrm{Q}_{16} / \mathrm{Q}_{14}$ & 35.79 & & 37.45 & & 43.17 & & 56.02 & & 62.94 & & 43.85 & & 49.56 & \\
\hline
\end{tabular}

